\title{
Registration of Organ Surface with Intra-operative 3D Ultrasound Image Using Genetic Algorithm
}

\author{
Ruoyun $\mathrm{Wu}^{1}$, Keck Voon Ling 2 , Wei Shao ${ }^{2}$, and Wan Sing $\mathrm{Ng}^{3}$ \\ ${ }^{1}$ Clinical Research Unit, Tan Tock Seng Hospital, 308433 Singapore \\ ${ }^{2}$ School of Electronic \& Electrical Engineering, Nanyang Technological University \\ 639798 Singapore \\ ${ }^{3}$ School of Mechanical \& Production Engineering, Nanyang Technological University, \\ 639798 Singapore
}

\begin{abstract}
The goal of this research is to register organ surface with intraoperative 3D ultrasound (US) image. The algorithm can be used to register preoperative images, such as MR and CT, with intra-operative US image, or to track organ surface in US image sequence. Simulation experiments were performed to demonstrate the registration of prostate surface with 3D US image in a population of 8 patients. Surfaces were segmented from US images and registered with the same images multiple times to show the accuracy and consistency. Surface tracking was demonstrated by registering the surface obtained from one US image with another US image of the same patient. Genetic Algorithm was used as the search engine to find out the 6-degree transform of the surface that best fit the US image gradients. The algorithm is observed accurate and achieved high consistency in all tested images with an average process time of 9.5 seconds.
\end{abstract}

\section{Introduction}

Diagnostic ultrasound (US) has been widely used to guide biopsy and minimally invasive treatments, due to its safety, low cost, and simplicity of use. Modern 3D US scanners can provide 3D volume data within a few seconds. However, the major drawback of US imaging is the poor image quality and resolution, compared to Magnetic Resonance (MR) and Computed Tomography (CT). Therefore, mapping the information obtained in MR and CT to US would enhance the intra-operative guidance capability of US. Tracking the organ surface in US image sequence is also helpful in diagnosis and robotics surgery.

\subsection{Review of Registration Methods for US Images}

There have been previous surveys of the medical image registration in the literature $[1,2]$. However, the registration of US images has not gained the same degree of development as other imaging modalities, probably due to the low signal-to-noise 
ratio of US images. Registration techniques generally fall into one of three categories: pixel-to-pixel (P2P), marker-to-marker (M2M), or marker-to-pixel (M2P). Most of the proposed approaches on registering US image to other image modalities fall into either P2P or M2M category.

$\mathrm{P} 2 \mathrm{P}$ approach compares the image intensities of both images, and registers according to mutual information or correlation ratio functions. Shekhar et al [3] used mutual information measurement, Pennec et al [4] and Roche et al [5] used correlation ratio, while Wachowiak et al [6] used both. P2P doesn't require explicit feature extraction and external tracking system, but is usually time-consuming.

M2M approach has two sub-categories: stereotactic-based and feature-based. In stereotactic-based approach, artificial markers are affixed to the patient prior to the pre-operative imaging session so that they are visible in the pre-operative images. During the operation, the physical positions of the markers and US probe are tracked by an external tracking system. Based on the relative position between the probe and the markers, the obtained 2D/3D US images can be registered to the pre-operative image. Gobbi et al [7] used optical tracking system, while Pagoulatos et al [8] used magnetic position senor. Stereotactic-based M2M approach could achieve very fast registration, as they do not need to process the image pixels. However, it is usually difficult to use artificial markers except in skull-base surgery.

Feature-based M2M approach extracts homologous features, such as bony landmark, organ surface, and vascular structure, in both pre- and intra-operative images and use these features as natural fiducial markers for registration. For pre-operative images, the features are usually extracted either manually or semi-automatically. For intra-operative images, the features are usually extracted automatically (or with minimal user intervention) to avoid process delay. Inoescu et al [9] extracted contours from intra-operative US image and registered them to the surface model reconstructed from pre-operative CT images. Porter et al [10] extracted major vessel volume from intra-operative color Doppler US image using color pixel-value threshold, and matched it to the volume reconstructed from pre-operative MR. The problem with feature-based M2M approach is that the automatic feature extraction from intraoperative US image is still not robust enough, and color Doppler may not work in cases where there are no major vessels being scanned.

M2P approach extracts features from pre-operative image, just like the featurebased M2M approach mentioned above. However, instead of extracting any features explicitly from the intra-operative image, M2P approach uses the pixel intensities or gradients directly. King et al [11] built statistical liver surface model from preoperative CT/MR images and registered the model directly to the intra-operative US images using Bayesian formulation. It is interesting to see that M2P itself is a special form of feature extraction for intra-operative images. Normal feature extractions only have very limited prior knowledge of the target feature. For example, the shape and size of the feature are usually assumed unknown. In M2P approach, however, rich information of the target feature, such as the shape and size of the organ surface, is available from the pre-operative image. Based on these prior knowledge, the features can be identified in the intra-operative US images in a much more robust manner, while the robustness is especially critical for US images. 


\subsection{Our Context and Approach Choice}

Our application is Robotic Prostate Biopsy, in which 3D US would be used to guide a medical robot to perform precise biopsy. As candidate prostate cancer sites are identifiable in MR images by using Magnetic Resonance Spectroscopic (MRS) technology [12], registering the surface extracted from pre-operative MR image to the intraoperative US images would also map these cancer sites.

In the context of our application: P2P is considered too slow; stereotactic-based $\mathrm{M} 2 \mathrm{M}$ is not suitable, as artificial markers cannot be used; feature-based M2M may not be robust and fast enough. Therefore, we chose M2P approach, using the prostate surface as a natural marker. In this paper, simulation experiments were performed to demonstrate the rigid registration of prostate surface with 3D US image using Genetic Algorithm (GA). Assuming that the prostate surface does not change significantly ${ }^{1}$ when under different scanning, our approach will be able to register pre-operative MR with intra-operative US, or track the prostate surface in US image sequence.

\section{Genetic Algorithm Based Registration}

Genetic Algorithm (GA) [13] is a general-purpose search engine inspired by biological evolution to find optimal "solutions" among an enormous number of possibilities. It is a massively parallel (global) search method: rather than work on one species at a time, it can test and change millions of species in parallel. Species are chromosomes that encode solutions to the problem at hand. GA processes require a fitness function that evaluates how well a chromosome solves the problem and assigns a fitness score to each chromosome accordingly. Species evolve by means of random variation (via mutation, recombination, and other operators), followed by natural selection in which the fittest tend to survive and reproduce, thus propagating their genetic materials encoded in chromosome to future generations.

GA has been proved as a robust algorithm to detect prostate boundaries in 2D US images [14]. In [14], a statistical model was used to specify the feasible shape, size and location of the prostate boundary for the GA search engine. GA then evolves a population of these $2 \mathrm{D}$ boundaries while computing their fitness score in the gradient map of 2D US image. In the 3D domain, the same idea could be extended to let GA evolves a population of 3D prostate surfaces with different location and orientation while computing the fitness degree of them in the gradient map of 3D US image.

\subsection{GA Chromosome Encoding}

Rigid registration of two subjects in 3D domain is to find out the optimal transform $T$ that fit them best. The transform can be parameterized with the 6 bound parameters,

1 Rectal US probe may slightly deform the posterior of prostate in US scanning. Similar deformation can be introduced in MR scan by using a rectal coil. 
three for translation and three for rotation. Therefore, our GA chromosome encodes these 6 parameters as its genetic materials. The range of translation parameters is based on the assumption that the geometry center of the surface should be within the volumetric image, while the range of rotation parameters covers all possibilities.

Unlike many other registration approaches, GA-based algorithm does not require any initial positioning of the subjects to be registered. In another words, when the GA is initialized, the first generation of chromosomes contains transforms that are generated randomly. Although using appropriate initial position may improve the performance by reducing the search range of the transform parameters, we didn't use one as getting a good initial position in our case may slow down the total processing time.

\subsection{Fitness Function for 3D US Images of Prostate}

It can be observed that the prostate appears in an ultrasound image as a relatively dark region surrounded by a bright halo, with subsequent dark regions. The surface locates at the border between the dark region and the bright halo, where the image gradient is high and pointing from outside into the prostate [14]. Therefore, the fitness score is calculated as the average image gradient on the transformed surface. Only the gradient components pointing to the inside of the transformed surface are considered.

Given the 3D image gradient map $G$ and $n$ points that equally spaced on the surface, the fitness function for transformation $T$ would be:

$$
f=\frac{1}{n} \sum_{k=1}^{n}\left(G\left(T\left(x_{k}\right)\right) \cdot T\left(N_{k}\right)\right)
$$

where $x_{k}$ is the point position and $N_{k}$ is the normal direction of the surface pointing from outside into the prostate. Note that $x_{k}$ and $N_{k}$ are using the surface's local coordinate system that uses the geometric center of the surface as the original point. If a surface point does not fall into the US scanning area, e.g., outside the image box, that point will be excluded from Equation 1.

To get equally spaced points on the surface, we extracted 20 equally spaced ISO curves from the prostate surface, 30 equally-spaced points were then obtained from the longest curve and less number of points from the other curves so that the intervals between the points on each curve are as equivalent as possible. Usually less than 300 surface points and their normal directions are passed to the GA engine. In order to get smoother gradient map, the US images are also Gaussian filtered beforehand.

\subsection{GA Strategy, Scheme, and Parameters}

The GA engine was implemented based on Galib 4.2 [15], which supports various chromosome encode schemes, evolution strategies, selection schemes, scaling scheme, crossover operators and mutation operators. We conducted a test similar to 
that in [14] for different combination of these schemes and strategies to compare their performance, and used the following in our application: real-value array chromosome encoding scheme, incremental GA evolution strategy with crowding replacement, tournament selection scheme, linear scaling scheme, uniform crossover operator and Gaussian mutation operator.

The GA parameter setting for our application is a population of 400 chromosomes goes for 300 generations, with $95 \%$ crossover rate and $1 \%$ mutation rate. Like in [14], it was found very easy to configure these GA parameters. The bigger the population size and generation number, the better the registration outcome in term of achieving higher fitness score, at the expense of longer processing time. The crossover rate and mutation rate does not affect the performance significantly, as long as they are round the fore-mentioned figures.

\section{Experiments and Results}

The 3D US images used in our experiments were taken from 8 patients using two commercial 3D US scanners (Voluson 530D and Combinson 430 from Medison). These images have various dimensions ranging from $212 \times 192 \times 248$ to $256 \times 256 \times 256$, with pixel sizes ranging from $0.173 \mathrm{~mm}$ to $0.274 \mathrm{~mm}$ per pixel.

The surfaces used in the experiments were reconstructed manually from a set of parallel slides from 3D US image. Tool has been developed to help the user to generate Non-Uniform Ration B-Spline (NURBS) curves from a few mouse-clicks for 2D images, and reconstruct NURBS surface by skinning over a stack of curves. Figure 1 illustrates the reconstruction of the prostate surface.
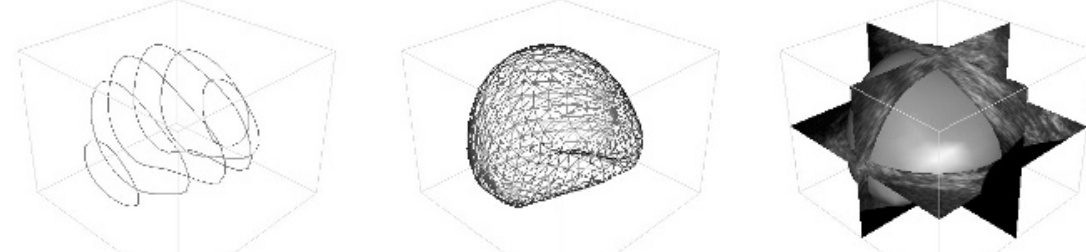

Fig. 1. Reconstructing a prostate surface from a stack of manually outlined closed NURBS curves. (a) Closed curves outlined by the user; (b) Reconstructed prostate surface wireframe; (c) Solid surface displayed with the 3D US image

\subsection{Register Prostate Surfaces with Their Source US Images}

In order to demonstrate the accuracy and consistency of the algorithm, we reconstructed prostate surface from US image, and then registered the surface with the source US image they are from. An expert manually reconstructed the prostate surfaces for 8 images, one image for one patient. Each surface was reconstructed using 
6 7 manually outlined NURBS curves, which implies that the reconstructed surface may not be precise due to the NURBS surface interpolation between these curves. However, we did this purposely to show that the registration algorithm could tolerate this minor difference between the reconstructed surface and the real surface.
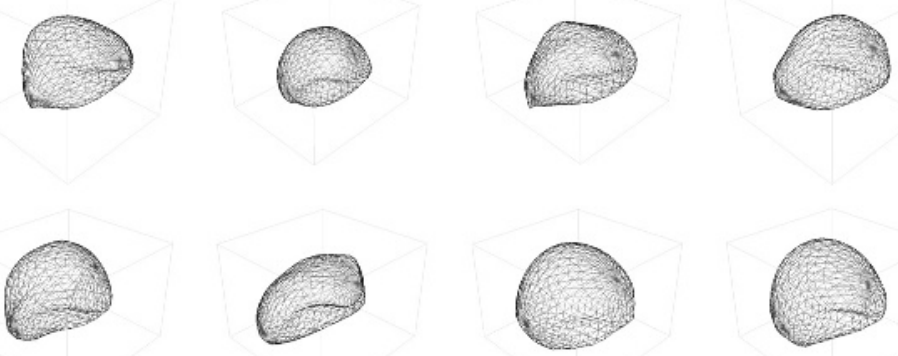

Fig. 2. The eight surfaces from different patients are used in the experiments.

The same algorithm settings were used for all images without any changes. The registration was done 10 times for each of the surface-image pairs. The errors in Table 1 show the location/orientation differences between the registered surface and the surface reconstructed by expert. The average error and maximum error indicates the accuracy, while the standard deviation of 10 runs indicates the consistency.

Table 1. Experimental results of registration in the population of 8 patients.

\begin{tabular}{|c|c|c|c|c|c|c|}
\hline \multirow{3}{*}{$\begin{array}{c}\text { Image } \\
\text { No. }\end{array}$} & \multicolumn{4}{|c|}{ Accuracy } & \multirow{2}{*}{\multicolumn{2}{|c|}{$\begin{array}{c}\text { Consistency } \\
\begin{array}{c}\text { Std. deviation of } \\
\text { errors }\end{array} \\
\end{array}$}} \\
\hline & \multicolumn{2}{|c|}{$\begin{array}{c}\text { Translation error } \\
(\mathrm{mm})\end{array}$} & \multicolumn{2}{|c|}{ Rotation error (degree) } & & \\
\hline & Avg. & Max. & Avg. & Max & Translation & Rotation \\
\hline 1 & 0.431 & 0.741 & 0.030 & 0.058 & 0.209 & 0.013 \\
\hline 2 & 0.486 & 0.873 & 0.040 & 0.069 & 0.227 & 0.021 \\
\hline 3 & 0.709 & 1.397 & 0.052 & 0.091 & 0.326 & 0.030 \\
\hline 4 & 0.409 & 1.060 & 0.039 & 0.106 & 0.265 & 0.029 \\
\hline 5 & 0.525 & 1.298 & 0.028 & 0.079 & 0.413 & 0.024 \\
\hline 6 & 0.386 & 0.599 & 0.035 & 0.077 & 0.158 & 0.020 \\
\hline 7 & 0.521 & 0.701 & 0.093 & 0.126 & 0.186 & 0.025 \\
\hline 8 & 0.633 & 0.854 & 0.042 & 0.092 & 0.179 & 0.024 \\
\hline Avg. & 0.513 & 0.940 & 0.045 & 0.087 & 0.245 & 0.023 \\
\hline
\end{tabular}

\subsection{Register Prostate Surface with Non-source Image}

To demonstrate tracking organ surface in 3D US image sequence, we segmented the prostate surface from one US image, and then register that surface to another image 
of the same patient. The registered surface perfectly matched the real surface in the new image from the expert's viewpoint.
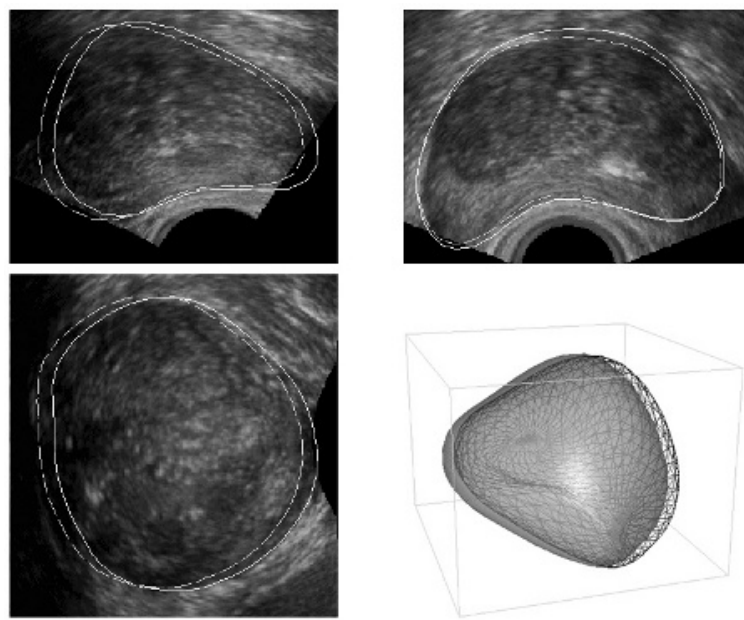

Fig. 3. Registering the prostate surface to a different US image of the same patient. The three images are orthogonal planes of the 3D volume data. The dashed line contours and wire-frame indicate the original location, while the solid line contours and solid surface are the registration result with the new image

Our application takes 7.5 10.5 seconds, with an average of 9.5 seconds, to complete the registration, running on a Pentium IV 2.4GHz PC with 512MB RAM, under J2SE 1.3.1 (GUI is written in Java) and Microsoft Windows 2000 Professional.

\section{Conclusions}

We have presented a new fully automated and fast method to rigidly register organ surface with 3D US image using Genetic Algorithm (GA). Testing was performed on several clinical data sets. The accuracy was evaluated by comparing the registered surface with the surface reconstructed by expert. We estimated the worst registration errors to be of the order of $1 \mathrm{~mm}$ for translation and 0.1 degree for rotation. The algorithm is also observed achieving high consistency in all tested images, showing the excellent robustness of the algorithm. The performance, including the average processing time of 9.5 seconds, is considered to be acceptable by the surgeons/radiologists involving in the Robotic Prostate Biopsy project. Further testing will be conducted when appropriate MR-US image pairs are available.

In the current implementation, the registration is done rigidly. But extending our algorithm to non-rigid registration is possible. Statistical surface model could be used to add in additional shape control parameters into the chromosome. Alternatively, a constrained local deformation could be made after a rigid best fit. 
Acknowledgement. This work was supported by National Science and Technology Board of Singapore and the Ministry of Education of Singapore, under JTARC 17/97, a strategic research grant, of which this project is a part.

\section{References}

1. Maintz J.B.A. and Viergever M.A.: A survey of medical image registration. Med. Image Anal. 2 1-36, 1998.

2. Derek L.G.H., Philipp G.B., Mark H. and David J.H.: Medical image registration. Phys. Med. Biol. 46 (2001) R1-R45, 2001

3. Shekhar, R., Zagrodsky, V.: Mutual information-based rigid and nonrigid registration of ultrasound volumes. IEEE Transactions on Medical Imaging, pp 9-22, vol. 21, 2002

4. Pennec, X., Ayache, N., Roche, A., Cachier, P.: Non-rigid MR/US registration for tracking brain deformations. Proceedings of International Workshop on Medical Imaging and Augmented Reality, pp 79-86, 2001

5. Roche, A., Pennec, X., Malandain, G., Ayache, N.: Rigid registration of 3-D ultrasound with MR images: a new approach combining intensity and gradient information. IEEE Transactions on Medical Imaging, pp 1038-1049 vol. 20, Issue: 10, 2001

6. Wachowiak, M.P., Smolikova, R., Elmaghraby, A.S.: Hybrid optimization for ultrasound and multimodal image registration. Proceedings of the 23rd Annual International Conference of the IEEE Engineering in Medicine and Biology Society. pp 2418-2421 vol. 3, 2001

7. Gobbi, D.G., Comeau, R.M., Lee, B.K.H., Peters, T.M.: Integration of intra-operative 3D ultrasound with pre-operative MRI for neurosurgical guidance. Proceedings of the 22nd Annual International Conference of the IEEE Engineering in Medicine and Biology Society, pp 1738-1740 vol. 3, 2000

8. Pagoulatos, N., Edwards, W.S., Haynor, D.R., Yongmin Kim: Interactive 3D registration of ultrasound and magnetic resonance images based on a magnetic position sensor. IEEE Transactions on Information Technology in Biomedicine, pp 278-288, 1999

9. Ionescu G., Lavallee S., Demongeot J.: Automated Registration of Ultrasound with CT Images: Application to Computer Assisted Prostate Radiotherapy and Orthopedics. Proc. MICCAI'99 Springer Lecture Notes in Computer Science 1679: 768-777, 2000

10. Porter, B.C., Rubens, D.J., Strang, J.G., Smith, J., Totterman, S., Parker, K.J.: Threedimensional registration and fusion of ultrasound and MRI using major vessels as fiducial markers. IEEE Transactions on Medical Imaging, pp 354-359, vol. 20, 2001

11. King, A.P., Blackall, J.M., Penney, G.P., Hawkes, D.J., Tracking liver motion using 3-D ultrasound and a surface based statistical shape model. IEEE Workshop on Mathematical Methods in Biomedical Image Analysis, pp. 145-152, 2001

12. Scheidler, J., Hricak, H., Vigneron, D. B., et al: Prostate cancer: localization with threedimensional proton MR spectroscopic imaging - clinicopathologic study, Radiology, vol. 213, pp. 473-480, 1999.

13. Mitchell M.: An introduction to genetic algorithm. MIT press, ISBN: 0262631857, Cambridge, Mass., MIT Press, 1996.

14. Wu R., Ling K.V., Ng W.S.: Automatic Prostate Boundary Recognition in Ultrasound Images Using Feature Model and Genetic Algorithm. Journal of Ultrasound in Medicine, Vol. 19, pp. 771-782, 2000.

15. Wall Matthew: GA-lib: a C++ library of genetic algorithm components, Mechanical Engineering Dept., MIT, 1996. 